Z. Klin. Chem. Klin. Biochem.

11. Jg. 1973 , S. $94-95$

\title{
International Nomenclature in Clinical Chemistry 1972
}

Modern prevention, diagnosis, and treatment of disease require numerous and sophisticated clinical chemical results.

In order to produce the necessary data, the laboratories employ qualified personnel of different education as well as specialized chemical and physical technology.

In order to evaluate the results clinicians must have some knowledge of chemistry and biochemistry.

Thus, clinicians and clinical chemists alike have to communicate with the basic sciences - albeit to a different degree and with different emphasis.

To facilitate intra- and interdisciplinary communication the International Organization for Standardization (ISO), the International Union of Pure and Applied Physics (IUPAP), the International Union of Pure and Applied Chemistry (IUPAC), and the International Union of Biochemistry (IUB), during the last years, have agreed on a common nomenclature, based on the International System of Units (SI). Already, technology and governments have started to approve and implement the recommendations.

Medicine, including clinical chemistry, cannot afford to remain outside this community of science by maintaining a separate out-dated language. This is the reason why the International Federation of Clinical Chemistry (IFCC) and the Section on Clinical Chemistry of IUPAC have issued recommendations in consonance with those of the abovementioned international bodies $(1,2,3)$.

Recently, most of the rules have been restated in an important Recommendation for Use of SI in Clinical Laboratory' Measurements 1972 of the International Committee for Standardization in Hematology, International Federation of Clinical Chemistry, and World Association of (Anatomic and Clinical) Pathology Societies (4). This Recommendation is published on page 93 of the present issue. It will also appear in a number of other clinical chemical, haematological, and clinical pathology journals.

\section{Main principles}

For details of nomenclature the original clinical chemical recommendations should be consulted. However, the outlines are as follows.

1. The units of measurement are those given by the International System of Units (5) - SI units. The base units, one for each basic kind of quantity, are listed in Table 1.
Tab. 1

Basic kinds of quantities and base units

\begin{tabular}{lll}
\multicolumn{1}{c}{$\begin{array}{c}\text { Basic kind of quantity } \\
\text { English name }\end{array}$} & \multicolumn{2}{c}{ SI Base unit } \\
\multicolumn{1}{c}{ English name } & Symbol \\
\hline length & metre & $\mathrm{m}$ \\
mass & kilogram & $\mathrm{kg}$ \\
time & second & $\mathrm{s}$ \\
electric current & ampere & $\mathrm{A}$ \\
thermodynamic temperature & kelvin & $\mathrm{K}$ \\
luminous intensity & candela & $\mathrm{cd}$ \\
amount of substance & mole & mol \\
\hline
\end{tabular}

Derived units are created by multiplication and division of base units, e. g. the coherent derived unit of pressure is $\mathrm{kg} /\left(\mathrm{s}^{2} \mathrm{~m}\right)$, also called pascal $(\mathrm{Pa})$. As regards units in enzymology the IUB/IUPAC Recommendations (6) apply. 2. The preferred unit of volume is 'litre', symbolized ' $l$ ', in accordance with chemical usage. The coherent SI unit is 'cubic metre', symbolized ' $\mathrm{m}^{3}$ ', but litre is more convenient and permitted.

3. The use of decimal multiples and sub-multiples of $S I$ units is facilitated by the series of SI prefixes; those having exponents of $3 n$, where $n$ is a whole negative or positive number, are preferred (Tab. 2).

Tab. 2

Decimal multiples and sub-multiples; SI prefixes

\begin{tabular}{llllll}
\hline & \multicolumn{2}{c}{ SI prefix } & & \multicolumn{2}{c}{ SI prefix } \\
Factor & Name & Symbol & Factor & Name & Symbol \\
\hline $10^{12}$ & tera & $\mathrm{T}$ & $10^{-3}$ & milli & $\mathrm{m}$ \\
$10^{9}$ & giga & $\mathrm{G}$ & $10^{-6}$ & micro & $\mu$ \\
$10^{6}$ & mega & $\mathrm{M}$ & $10^{-0}$ & nano & $\mathrm{n}$ \\
$10^{3}$ & kilo & $\mathrm{k}$ & $10^{-12}$ & pico & $\mathrm{p}$ \\
& & & $10^{-18}$ & fernto & $\mathrm{f}$ \\
& & & $10^{-18}$ & atto & $\mathrm{a}$ \\
\hline
\end{tabular}

4. In a given base or derived unit only one prefix should be used. If the unit is a fraction the prefix, preferably, is put in the numerator; an exception is the base unit kilogram. Thus, $\mathrm{m} \mu \mathrm{m} \rightarrow \mathrm{nm}, \mu \mathrm{mol} / \mathrm{ml} \rightarrow \mathrm{mmol} / \mathrm{l}$, and $\mu \mathrm{g} / \mathrm{g} \rightarrow \mathrm{mg} / \mathrm{kg}$. Note that the volume base of concentration units should be the litre.

5. For quantities comprising components with sufficiently well known chemical structure it is recommended to employ those with kinds of quantities of a 'molecular nature', i. e. based on 'amount of substance' with the unit 'mole'. There is more biochemical and, thus, pathobiological information in stating, e. g., the substance concentration of albumin in serum than in giving its mass concentration: The substance concentration in serum of bilirubins directly shows a useful relationship to that of albumin. 
6. The name of a type of quantity should always contain information on 1) system, 2) component, and 3) kind of quantity; the result is a product of 4) numerical value, and 5) unit. The actual form of the statement may be a sentence like the substance concentration of sodium ion in serum was 142 millimoles per litre' or a set of conventions may' lead to, e. g., 'S-Sodium ion, substc. $=142 \mathrm{mmol} / \mathrm{l}$.

7. The complete answer should contain or refer to sufficient information on method in its widest sense (including state of patient), error, reference values ('normal values'), and comments - as well as administrative data.

\section{Conclusion}

The implementation of some of the above recommendations will result in some initial inconveniences. Practical experience from 'converted' countries like the Netherlands, Finland, and Denmark has shown that the problems are surmountable if the change is preceded and followed by education of all types of involved personnel - and occurs with mutual cooperation.

The advantages of the new system are the easier access to other branches of pure and applied science, more useful information, and the eventual substitution of current diversity in expression with a single form for presentation of clinical chemical data.

\section{References}

1. Dybkaer, R. \& Jørgensen, $K .$, Quantities and Units in Clinical Chemistry. Including Recommendation 1966 of the Commission on Clinical Chemistry of the International Union of Pure and Applied Chemistry and of the International Federation for Clinical Chemistry. Mungsgaard, Copenhagen 1967. Pp. $x+102$. 2. International Union of Pure and Applied Chemistry and International Federation of Clinical Chemistry, Quantities and Units in Clinical Chemistry. Information Bulletin No. 20, February 1972. Pp. 24. - 3. International Union of Pure and Applied Chemistry and International Federation of Clinical Chemistry,
List of Quantitics in Clinical Chemistry. Information Bulletin No. 21, February 1972. Pp. 24. - 4. International Committee for Standardization in Hematology, International Federation of Clinical Chemistry, and World Association of (Anatomic and Clinical) Pathology Societies: Recommendation for Use of SI in Clinical Laboratory Measurements. Munich 1972, this J. 11, 93. - 5. National Physical Laboratory, SI The International System of Units. Her Majesty's Stationery Office, London 1970. Pp. $45+$ xi. - 6. Commission on Biochemical Nomenclature: Enzyme Nomenclature. New Edition to appear 1973.
Dr. R. Dybkaer

Dept. Clinical Chemistry

Geriatric Unit, De Gamles By

Nørre Alle 41

DK-2200 Copenhagen N 